\title{
Problèmes de la recherche sur le terrain dans les pays musulmans ${ }^{1}$
}

Depuis les travaux de Malinowsky, dans les années 20, il n'est plus concevable, en dépit de quelque exemple fameux, d'être ethnologue sans être ou avoir été ethnographe. Cette nécessaire participation à la vie des populations étudiées est l'un des traits qui distingue l'ethnologie des autres sciences humaines, elle en fait l'originalité tant au niveau épistémologique qu'au niveau pratique.

Sur le plan épistémologique, le travail de terrain, l'observation participante, implique un tel engagement personnel du chercheur que l'ethnologie ne peut en rien se targuer du semblant d'objectivité qui fonde la crédibilité des autres sciences sociales.

Sur le plan pratique, le type de relations qu'entretiennent sujet et objet de la recherche est déterminé par l'un et l'autre terme. C'est dire, pour ce qui est des déterminations dues à l'objet, que l'ethnographie d'une société islamique aura des thèmes et des manières différents de celle d'unę société amérindienne. Quant aux détermination dues au sujet, elles impliquent que chaque ethnologue a une vision des choses partielle et propre. C'est dire que les pages qui suivent n'engagent que moi, que l'accord ou le désaccord d'un autre ethnologue ne suffit pas à accréditer ou discréditer les idées que j'émet.

Traditionnellement préocupée avant tout des «Naturvölker", l'ethnologie ne s'est intéressée que tardivement aux sociétés de l'Islam oriental. L'Islam africain, fait de cultures moins «raffinées» et surtout colonisées par les puissances européennes, fut dès le XIXe siècle un terrain d'enquêtes ethnographiques. Dans le même temps en revanche, ceux que l'on nommait orientalistes étaient avant tout historiens, philologues ou philosophes. Il faut attendre le XXe siècle pour voir paraître les premières études ethnologiques consacrées à des populations de l'Orient musulman. Dans ce développement récent les travaux se sont regroupés autour de quelques thèmes privilégiés.

L'extension actuelle de l'Islam recouvre la plus importante des zones du nomadisme pastoral; ce nomadisme est donc l'un de ces thèmes, privilégié surtout depuis les années 60 , dans les approches d'écologie culturelle et d'anthropologie politique.

Quant aux communautés rurales de l'Orient, elles ont fournit le modèle d'un mode de production particu-

1 J'ai bénéficié, pour la rédaction de cet article, des conseils du Pr. $P$. Centlivres que je tiens à remercier. lier. Les remises en question de ce «mode de production asiatique" sont depuis vingt ans l'occasion de nombreuses études et d'intérêts nouveaux pour des pays qui ont connu dans l'histoire de grands systèmes d'irrigation.

Je me référerai surtout au cadre du monde de la culture iranienne, pour aborder les problèmes que pose aux ethnologues la recherche de terrain en pays musulman.

La position de l'ethnographe, dans un tel terrain, est d'abord celle d'un étranger, c'est à dire d'un non musulman auquel s'applique parfois le terme injurieux de käfer: païen. Une telle attitude est historiquement fondée: durant plusieurs siècles, le règlement de la dhimma, attribué au calife Omar, fixa le statut des étrangers au sein de la communauté musulmane. Ce système est, en droit, tombé en désuétude, mais il a profondément marqué la mentalité populaire et il subsiste dans la pratique par le poids de traditions devenues des habitudes de comportement.

Avant le calife Omar, le Prophète avait signé en faveur des chrétiens de la région du Nejran un pacte soucieux de respect et d'égalité entre chrétiens et musulmans. L'institution de la dhimma, en revanche, implique une inégalité fondamentale. Le chrétien ayant accepté de passer avec la communauté musulmane un pacte d'allégeance ('ahd) recevait en retour une "protection tutélaire» (dhimma) qui faisait de lui un sujet de cette communauté et le plus souvent, un citoyen de seconde zone. Pour être plus précis, il faut spécifier encore que le statut de dhimmi ne pouvait être accordé qu'aux "gens du livre»: chrétiens, juifs, et sabéens. Les idolâtres ou tous ceux qui ne reconnaissaient pas l'unité de Dieu, n'avaient, en droit, pas de place dans la cité musulmane.

Quant aux dhimmi, si leurs cultes et leurs usages étaient respectés, il n'en étaient pas moins soumis à un certain nombre de devoirs restrictifs: obligation de payer tribut, interdiction d'épouser une musulmane, d'édifier de nouvelles églises, de construire une maison plus haute que celle du voisin musulman. Des obligations vestimentaires imposaient aux "gens du livre» des signes distinctifs de couleurs différentes selon la religion, dans lesquels on a pu voir l'origine de l'étoile jaune des juifs de l'Allemagne nazie.

François-Xavier Meyer, lic. ès 1., Fontaine-André 26 , 2002 Neuchâtel 
Mais à en croire Gardet et Massignon, il ne faudrait pas s'en tenir à l'apparente sévérité de ce règlement. D'abord, l'application de la dhimma varie selon les lieux et les périodes, ensuite ce statut serait, à de nombreux égards, préférable à celui des "hérétiques» dans les communautés européennes du Moyen Age, enfin, il faut y voir de la part de la communauté musulmane et dans son optique, une générosité qui participe du droit d'asile et de l'hospitalité sacrée. Ainsi le dhimmi devrait être considéré comme un hôte permanent et, pour suivre Gardet, il est normal que l'hôte ne participe qu'avec discretion à la vie de ceux qui le reçoivent. L'hospitalité sacrée dont il vient d'être question est en effet l'un des traits marquants de l'attitude musulmane face à l'étranger. Une dizaine de versets du Coran prescrivent la conduite à tenir envers les voyageurs: bonté, générosité, protection. Le Livre (c'est là le nom du Coran) reprendrait ainsi une partie de la tradition des tributs bédouines d'Arabie. Or ces tribus n'accordaient le droit d'asile que durant trois jours, après quoi l'hôte devait quitter la tente et le camp hospitalier. (Gardet, 1961: 78)

Dans l'Afghanistan actuel il est de bon ton, particulièrement chez les Pashtun du sud-est, d'offrir une hospitalité illimitée, d'insister même sur le fait que le séjour peut durer toute une année. L'hôte s'offre toujours à satisfaire tous les désirs de son invité, il le sert respectueusement en se tenant en-dehors du tapis, il lui offre les meilleures nourritures disponibles dans la maison. Mais un tel système d'hospitalité n'est possible qu'à deux conditions minimales: une réciprocité, directe ou indirecte, qui permette à l'hôte d'être à son tour l'invité; une connaissance et un respect, de la part de l'invité, des limites hors desquelles il est pour son hôte un fardeau.

De par sa situation de voyageur, loin de chez lui,l'ethnologue n'est souvent pas à même de participer au système de réciprocité. Il devra donc compenser ce handicap. Les cadeaux sont un moyens de compensation, mais l'hospitalité étant pour l'hôte un devoir religieux, le cadeau ne sauvait être une contrepartie. Il faudra donc procéder par détour, faire le cadeau à un enfant, à un serviteur pauvre. Quant au respect des limites, ce n'est qu'avec l'expérience du terrain et une sensibilité attentive que l'invité peut apprendre à donner à ses hôtes autant de satisfactions qu'il en reçoit. En toute bienséance, l'invité ne devrait s'attarder que le temps nécessaire à la poursuite de son voyage et si la maladie ou le mauvais temps ne le contraignent pas, c'est en général le lendemain qu'il quittera la tente ou la maison qui l'a hébergé. Or, pour satisfaire aux besoins de son enquête, l'ethnologue devrait faire sur le terrain les plus longs séjours possible. En d'autres cas également, les règles de l'hospitalité et les besoins du travail divergent.

Ainsi l'ethnologue ne sera un hôte parfait qu'à la condition d'oublier pour un instant les buts de son séjour et les impératifs de son enquête L'invité ne devrait en effet s'occuper de la vie de son hôte que dans la mesure où celui-ci le veut bien. Or l'ethnologue cherche, par profession, à en savoir toujours plus. Il se heurte ainsi à différentes barrières qui protégent dans l'Islam l'individu et sa famille.

De telles barrières ne sont jamais uniformément définies. Elles varient d'une contrée, d'une occasion à l'autre. L'ethnologue nouveau venu n'aura pas trop de toute son attention pour apprendre et pressentir rapidement ces limites fluctuantes, pour ne pas commettre d'impair tout en obtenant un maximum d'informations. Dans cet apprentissage des interdits, celui qui concerne les femmes se remarque d'entrée.

Haram désigne en arabe à la fois le sacré et l'interdit. Le harem est dans la maison arabe la partie réservée aux femmes.

L'index du Coran dans l'édition Pléïade donne sous le mot femme plus de 170 références!

L'interprétation de l'ensemble de ces versets est l'occasion de nombreux débats chez les spécialistes de l'Islam. Les novateurs qui veulent changer le statut de la femme dans le monde musulman y trouvent leur compte, leurs adversaires conservateurs également.

Je n'entrerai pas dans le débat, m'en tenant aux implications pour l'observateur, de la situation actuelle de la femme dans le monde de la culture iranienne et surtout en Afghanistan.

L'architecture des maisons musulmanes sépare le plus souvent la chambre d'hôte du reste de la maison, domaine où les femmes circulent librement. De fait, l'hôte ne devrait en aucun cas s'intéresser aux femmes. S'il a cependant quelque raison de parler d'elles il ne les désignera pas explicitement mais parlera de la «mère des enfants», "la maisonnée» ou même seulement «les enfants». Si cet hôte devient un habitué du lieu et qu'on veuille lui faire l'honneur de le considérer comme "adopté», il aura peut-être l'occasion de voir la maîtresse de maison, il recevra peut-être de la main des hommes des cadeaux préparés à son intention par les femmes, mais ce n'est qu'après une longue approche, qu'il pourra, peut-être, parler librement avec les femmes de la maison.

La femme musulmane est en effet, en principe, coupée de tout contact avec d'autres hommes que ceux de la famille proche. En Afghanistan, la bourgeoise ne sortait dans la rue que couverte d'un voile qui la dissimulait entièrement, et ce n'est guère que dans la capitale que cette coutume cesse d'être toujours en vigueur. Telle femme d'une famille de ma connaissance est sortie de sa maison deux fois en trois ans. L'homme à qui s'adresse d'abord l'étranger se doit de protéger les femmes de sa famille de tout contact avec cet étranger.

Qu'en est-il alors de cette barrière vis-à-vis d'une femme ethnologue? On pourrait s'attendre à ce que les contacts entre femmes soient plus faciles; A. Tual (1976: 116) écrit cependant:

"Les villageois demandaient de «bien vouloir comprendre qu'ils leur semblaient difficile que nous puissions demander de voir les femmes du village, leurs femmes.» 
Les mêmes villageois se proposent de remplacer leurs femmes pour répondre aux questions qui concernent ces dernières et ce n'est que quand ils se trouvent incapables de répondre qu'ils décident enfin de permettre le contact entre leurs femmes et A. Tual.

Mais dans le cas ci-dessus, A. Tual n'est femme que biologiquement; socialement, elle est un être hybride: $\mathrm{Ni}$ mère, ni épouse, ni épousable, voyageant de son propre chef, s'adressant ouvertement aux hommes, elle occupe en tant qu'ethnologue une position d'homme. Il est alors normal qu'on la tienne d'abord à l'écart des femmes. Passées les premières approches, l'ethnologue femme pourra cependant jouer de ces ambiguïtés sexuelles et enquêter aussi bien chez les hommes que chez les femmes. Si toutefois cette enquêteuse est accompagnée d'un interprète masculin, c'est à nouveau les barrières mises à ce dernier qui limiteront le domaine de l'enquête.

Il n'y a pas que le sexe de l'ethnologue pour déterminer les rapports avec ses informateurs musulmans. Ainsi les hommes mariés auront une position préférable à celle des célibataires. L'homme marié, même s'il voyage seul, est plus proche d'un père de famille afghan qu'un célibataire, et plus rassurant. Le premier a, dans son monde, des contacts avec les femmes, sait ce qu'est une famille, on lui répond alors avec la conscience d'une égalité respectueuse, on lui dit "padar jän»: père.

L'ethnologue célibataire en revanche ne peut-être que mister ou saheb (monsieur), purupaïsár (professeur) ou musăfer (voyageur). Il doit être tenu plus strictement qu'un père à distance des femmes. Son célibat, s'il n'est pas de prime jeunesse, sera regardé comme étrange: "Tu as de l'argent pour voyager, pourquoi est-ce que tu n'es pas marié?» Tel médecin du nord afghan, toujours habillé à l'européenne et ayant quelques connaissances d'anglais, s'excusant de ne m'inviter chez lui que pour un petit déjeuner, me promis que quand je le visiterais avec ma femme, je resterais chez lui plusieurs jours et ferais connaissance avec tous les membres de sa famille. Ce qui signifiait clairement, puisque je connaissais déjà les hommes, que j'approcherais alors également les femmes.

Dans tout l'Islam, le célibat, passé un âge décent et dans une situation sociale qui permet le mariage, est regardé comme une tare. Mahommet aurait dit que "L'haleine d'un homme marié est plus agréable à Dieu que la prière de soixante célibataires.» (De Castries, 1912: 117); Gauthier (1955: 46) qualifie le célibat du musulman d'«impiété». La polygamie implique que le célibat des femmes est plus rare que celui des hommes.

Il faut noter cependant que le clivage islamique entre société féminine et société masculine est généralement plus fort chez les sédentaires, citadins surtout, que chez les nomades. De part les conditions d'habitation, de part sa plus grande part aux activités de production également, la femme nomade ne peut être aussi recluse que la bourgeoise citadine. De plus, des traditions pro- bablement anté-islamiques, assurent à la femme chez les grands nomades du sud-est de l'Afghanistan une indépendance de langage et de mœurs, une participation à la vie de la communauté beaucoup plus grande que celle des citadines. Chez les nomades sahariens, la propriété des troupeaux de moutons et de chèvres assurent aux femmes une place d'importance dans la vie sociale.

Le sexe de l'ethnologue et son état civil ne déterminent pas que socialement son domaine d'activité: c'est aussi la géographie de cès activités qui s'en trouve affectée. Si la seule maison est l'espace des femmes ${ }^{1}$, l'espace des hommes s'organise autour de trois points principaux: champs ou pâture, mosquée, bazar.

C'est au bazar que les contacts se nouent le plus facilement entre les hommes. C'est là aussi que l'ethnologue masculin trouvera facilement des informateurs momentanément disponibles à un premier contact.

$\mathrm{Au}$ début des enquêtes régionales, avant que ne se nouent des relations individuelles, c'est dans la cour de la mosquée que l'enquêteur de passage rencontrera le plus souvent les villageois, ou dans la tente d'hôte chez les nomades ${ }^{2}$. L'intérieur de la mosquée est lui, dans la plupart des cas, un endroit interdit au visiteur non musulman. Dans cette cour de mosquée, les conversations libres entre ethnologue chrétien et indigène musulman ont souvent la religion pour thème. Tout musulman sait l'existence de nos évangiles, connaît Marie sous le nom de Maryam, Jésus sous le nom de Isa et tous les grands personnages de l'Ancien Testament. Comme un bon musulman connait sa religion beaucoup mieux que la majorité des occidentaux, l'ethnologue est souvent embarrassé pour répondre aux nombreuses questions.

Généralement de telles discussions conduisent immanquablement au thème de la conversion. Il ne paraît guère possible au premier abord et à en croire les musulmans eux-mêmes, de partager la vie de populations islamiques tout en restant chrétien. De fait, quelques uns se sont convertis à l'Islam. Conversions authentiquement religieuses dans certains cas, conversion à but pratique le plus souvent, mais le problème est moral et il ne m'appartient pas de juger.

Les conditions de chaque terrain sont particulières, l'invitation à se convertir va de l'ultimatum à la plaisanterie.

Quant à moi, je me suis vu pratiquement interdire une enquête par un dignitaire musulman qui m'avait été désigné comme le "chef» des nomades de la région. Revoyant ce mawlawi près de deux ans plus tard, ses

\footnotetext{
${ }^{1}$ Les femmes de cultivateurs participent tout de même aux travaux des champs, particulièrement au moment des récoltes.

${ }^{2}$ Dans le monde iranien, l'Islam se divise entre les sunnites qui reconnaissent les successeurs de Mahomet dans leur ordre historique, et les shiites pour qui Ali auraît dû succéder à Mahomet. Pour l'expérience que j'en ai en Afghanistan, l'étranger est traité différemment par les uns et par les autres, les shiites me semblant plus libéraux: dans un premier terrain fait en célibataire, je n'ai eu de contact avec les femmes que dans des familles shiites; les seuls shiites m'ont invité à assister aux cérémonies dans une mosquée (takiaxāna).
} 
premières paroles, immédiatement après les salutations, furent de me demander si je m'étais converti entretemps. ${ }^{1}$

Outre le problème pratique que pose une telle situation, elle soulève la question de la distance dans l'observation participante. l'ethnographie telle qu'elle est actuellement conçue, fait de la société qu'elle étudie un objet. Or cette objectivation ne peut se faire sans une distance minimum qui n'est pas compatible avec une assimilation totale dans le groupe étudié.

Quelques cas sont connus où le chercheur a totalement adopté le mode de vie d'une tribu. Ce faisant il a abandonné son bagage d'occidental, et ses outils d'ethnologue.

Mais l'autre extrême également empêche l'ethnologie. Pour se rapprocher de ses informateurs, pour accéder aux points d'observation les mieux situés, l'ethnologue devra adopter partiellement la conduite de ses hôtes: politesse, maintient, régime alimentaire sont considérés comme un minimum, mais ici encore l'importance de l'adoption est une affaire individuelle.

S'il n'est pas possible de tracer le profil idéal d'un ethnologue en pays musulman, du moins peut-on en donner quelques éléments.

Le travail en couple est la formule la plus adéquate à tous points de vue: le couple est une unité plus facilement intégrable par les indigènes que les célibataires, il permet des relations avec le monde féminin comme avec le monde masculin, il permet aussi aux enquêteurs un soutien mutuel. Ceux qui ont enquêté seuls pendant de longues périodes savent le besoin d'un tel soutien. Si le couple est accompagné d'enfants il sera encore plus facilement intégrable, ses contacts seront encore plus diversifiés.

Dans les pays musulmans, l'âge donne droit à une considération qui augmente la qualité des contacts. F. Balsan, explorateur des contrées les plus reculées du Baluchistan attribue à son grand âge de s'être sorti de situations périlleuses.

Mais la qualité la plus nécessaire à mon sens, dans ces pays où le temps ne compte pas, où l'achèvement temporel est moins valorisé que le spirituel, où la fièvre d'action et d'information de l'occidental paraît bien vaine à qui invoque la toute puissance d'Allah, c'est la patience qui seule permet le respect des interlocuteurs.

\section{Bibliographie}

BALSAN, FRANÇOIS: Etrange Baluchistan. Soc. Continentale d'Editions Modernes Illustrées; Paris, 1969; $360 \mathrm{p}$.

CHELHOD, JOSEPH: Introduction à la sociologie de l'Islam. Ed. Besson-Chantemerle; Paris, 1958;230 p.; index, bibliographie.

DE CASTRIES, HENRI : L'Islam. Ed, A. Colin; Paris, 1912; $350 \mathrm{p}$.; index.

GARDET, LOUIS: La cité musulmane. Librairie J. Vrin; Paris, 1961; 416 p.; index, bibliographie.

GAUTHIER, E. F.: Moeurs et coutumes des musulmans. Ed. Payot; Paris, 1955; 300p.

HADDAD, J.: Problèmes posés par la rencontre des culture islamique et occidentale. In: CrVILISATIONS, volXII,No3, 1962: 311-325.

TUAL, ANNY: Le statut féminin et l'usage de la parole. In: Studia Iranica, t. 5, fasc. 1, 1976:115-128.

\footnotetext{
${ }^{1}$ Il ne faudrait cependant pas conclure de là à une attitude générale des
} dignitaires musulmans. 